\title{
70\% Trichloroacetic Acid in the Treatment of Facial Sebaceous Hyperplasia
}

\author{
Anca Chiriac ${ }^{1,2,3}$, Piotr Brzezinski ${ }^{4}$, Adrian Năznean ${ }^{5}$, Cristian Podoleanu ${ }^{6}$, Simona Stolnicu ${ }^{7}$ \\ 1 Nicolina Medical Center, Department of Dermatology, Iași, Romania \\ 2 Apollonia University, Iași, Romania \\ 3 "P. Poni” Research Institute, Romanian Academy, Iași, Romania \\ 4 Department of Dermatology, 6thMilitary Support Unit, Ustka, Poland \\ 5 Department of Foreign Language, University of Medicine and Pharmacy, Tîrgu Mureș, Romania \\ 6 Department of Internal Medicine, University of Medicine and Pharmacy, Tîrgu Mureș, Romania \\ 7 Department of Pathology, University of Medicine and Pharmacy, Tîrgu Mureș, Romania
}

\section{CORRESPONDENCE}

Cristian Podoleanu

Str. Gheorghe Marinescu nr. 1

540099 Tîrgu Mureș, Romania

Tel: +40744573784

E-mail: podoleanu@me.com

\section{ARTICLE HISTORY}

Received: June 1, 2018

Accepted: July 29, 2018
Anca Chiriac • Str. Hatman Sendrea nr. 2, 700613 lași, Romania. Tel: +40 332808703

Piotr Brzezinski • Department of Dermatology, 6th Military Support Unit, os. Ledowo 1N, 76-270 Ustka, Poland. Tel: +48 692121516

Adrian Năznean • Str. Gheorghe Marinescu nr. 38 540139 Tîrgu Mureș, Romania. Tel: +40 265215551

Simona Stolnicu • Str. Gheorghe Marinescu nr. 38 540139 Tîrgu Mureș, Romania. Tel: +40 265215551

\section{ABSTRACT}

The present paper highlights the usefulness of 70\% trichloroacetic acid in treating sebaceous hyperplasia in elderly patients. Esthetics are an important issue, and different therapeutic modalities can be used, such as systemic isotretinoin, surgical excision, electrocautery, cryosurgery, topical photodynamic therapy and laser, but all these methods are expansive and invasive procedures that may result in scars, which are more extensive than the original lesions.

Keywords: $70 \%$ trichloroacetic acid, sebaceous hyperplasia, treatment

Sebaceous hyperplasia is a common benign proliferation of the sebaceous glands in middle-aged and elderly people. The clinical picture is striking and with great esthetic impact. Soft yellow papules with central umbilication are noticed not only on the face (particularly on the forehead, cheeks, and nose), but also on the genitalia, areola, and chest. Although they are known as senile sebaceous hyperplasia due to predominance in the elderly, nowadays sebaceous hyperplasia has been observed in middle-aged and even young women. In the majority of cases, the evolution is progressive, over several years, with crops of new lesions while the older ones become larger, umbilicated, and may discharge sebum from the center. No spontaneous clinical involution has been noted.

Sebaceous hyperplasia lesions can have variable morphological distribution: diffuse, unique large form, linear or zosteriform distribution, or along Blaschko's lines. In daily practice, most cases show a diffuse facial distribution of sebaceous hyperplasia papules.

Skin biopsy is not necessary every time; the diagnosis is usually clinical, although a histological report would confirm a lobular array of well-differentiated 


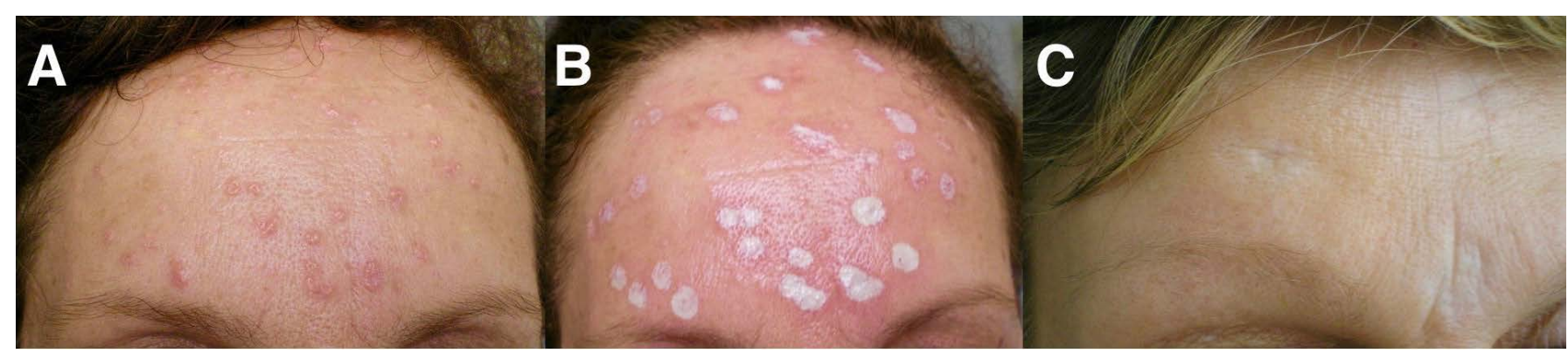

FIGURE 1. A - Adult sebaceous hyperplasia; B - The same patient with frosting of the skin during application of $70 \%$ trichloroacetic acid (third application); $\mathbf{C}$ - Close view of the frontal area of the same patient after 6 months of treatment

mature sebaceous lobules. In the pathologist's view on microscopic examination, sebaceous hyperplasia is defined only if at least 4 sebaceous lobules are attached to the infundibulum of the pilosebaceous unit, and sebocytes are filled with lipids.

The explanation why some individuals are predisposed to develop sebaceous hyperplasia is not completely understood, although different etiopathogenic factors have been incriminated such as natural aging, prolonged exposure to UV radiation, genetic predisposition. Presenile (or premature) diffuse familial sebaceous hyperplasia is a rare disorder in adolescents with family traits.

Molecular data support the hypothesis that the sporadic form may be a benign neoplasm instead of sebaceous hyperplasia; a pathogenic role of the EGFR-RAS-MAPK pathway has been demonstrated in sporadic sebaceous gland hyperplasia. ${ }^{1}$

During recent years, reports of other factors inducing sebaceous hyperplasia have been published. Cyclosporine administered in organ transplant recipients, especially renal transplant, was associated with the development of multiple sebaceous hyperplasia treated with oral isotretinoin. Additionally, sebaceous hyperplasia was reported in immune-suppressed organ transplant recipients in $16 \%$ of cases.

Various treatment methods have been attempted during the last decades, such as electrodessication, light lasers, applications of acids and photodynamic therapy, with different results and with the risk of scars and residual hyperpigmentation. Moreover, these methods are costly and difficult to access for patients. Oral isotretinoin is welldocumented in treating sebaceous hyperplasia; its action is based on inhibiting the size and function of sebaceous glands, but encumbered by serious side effects and recurrences upon discontinuation.

Monthly application of $70 \%$ trichloroacetic acid (TCA) for 5 consecutive months on the forehead of a 67-year-old woman diagnosed with sebaceous hyperplasia proved to be a valuable therapeutic option in our daily practice, with no side effects. Close follow-up of the patient showed no recurrence; new papules were treated in a similar way with positive results (Figure 1, panels A-C).

Based on these results, the method was applied to other patients with the same good tolerability and outcome. No laboratory investigations or other examinations were necessary during the topical treatment. Between sessions, an emollient cream with high UV protection index and avoidance of sun exposure were recommended.

According to the definition, TCA is a chemical peel that induces exfoliation of the skin. Initially, it was used for the cosmetic improvement of photo aging; subsequently, it became a versatile topical agent with a wide spectrum of clinical indications, exceeding its cosmetic purposes. In different concentrations with or without other ingredients, TCA was used in diverse pathologies, for example: striae rubra, plantar callus and warts, cutaneous leishmaniasis scars, for chemocauterization of small tracheocutaneous fistula, cervical intraepithelial neoplasia (CIN), acne, pyogenic granulomas in children, sebaceous hyperplasia. ${ }^{2}$

TCA is very caustic and should be handled with care. At a concentration of $70 \%$, it acts as a deep peel at the level of mid reticular dermis. ${ }^{3}$ A short contact produces the coagulation of epidermal and dermal proteins, as well as the necrosis of collagen from the upper reticular dermis. ${ }^{4}$

The necrotic layers are replaced with "seemingly normal skin" due to re-epithelization from the surrounding islets of keratinocytes and from skin appendages. ${ }^{5}$

TCA is a self-neutralizing peel, no systemic absorption has been reported, and patients do not complain of pain and/or pruritus. A burning sensation is sometimes described, but for a short period of time.

In conclusion, chemical cauterization with $70 \%$ TCA is a safe, easy-to-perform, non-expansive, and effective method for the treatment of sebaceous hyperplasia in patients to whom other methods are inaccessible. 


\section{CONFLICT OF INTEREST}

Nothing to declare.

\section{REFERENCES}

1. Groesser L, Singer S, Peterhof E, et al. KRAS, HRAS and EGFR Mutations in Sporadic Sebaceous Gland Hyperplasia. Acta Derm Venereol. 2016;23:737-741.

2. Simmons BJ, Griffith RD, Falto-Aizpurua LA, Bray FN, Nouri K. International League of Dermatological Societies; European Dermatology Forum Light and laser therapies for the treatment of sebaceous gland hyperplasia a review of the literature. J Eur Acad Dermatol Venereol. 2015;29:20802087.

3. Mradula PR, Sacchidanand S. A Split-face Comparative Study of $70 \%$ Trichloroacetic Acid and $80 \%$ Phenol Spot Peel in the Treatment of Freckles. J Cutan Aesthet Surg. 2012;5:261-265.

4. Lee JB, Chung WG, Kwahck H, Lee KH. Focal treatment of acne scars with trichloroacetic acid: chemical reconstruction of skin scars method. Dermatol Surg. 2002;28:1017-1021.

5. McCulloch EG, Langsdon PR, Maloney BP. Chemical peel with phenol. In: Roenigk RK, Roenigk HH, eds. Dermatologic Surgery, Principles and Practice. 2nd ed. UK/Oxford: Marcel Decker Ltd; 1996, p. 1147-60. 